\section{Funding for Antimicrobial Stewardship Programs: A Customizable Business Case Template}

Antimicrobials are a societal resource, and their future utility requires appropriate usage in the present. Successful antimicrobial stewardship programs (ASPs) in Canada and elsewhere have led to decreases in the incidence of Clostridium difficile infections and in the prevalence of colonization with resistant bacteria. ${ }^{1,2}$ Moreover, in institutions with an ASP, appropriate utilization of antimicrobials increases, which leads to cost savings. ${ }^{3-7}$ Although establishment of an ASP is a Required Organizational Practice of Accreditation Canada, ${ }^{8}$ antimicrobial stewardship is a poorly filled niche in health care in Canada. Challenging aspects of establishing an ASP relate to funding and long-term support from the hospital or health region administration. One of the biggest stumbling blocks to obtaining funding is the need to write a cogent, understandable business case for health care administrators.

Pharmacists are recognized by the International Pharmaceutical Federation ${ }^{9}$ and Accreditation Canada ${ }^{8}$ as key players in the management of antimicrobial resistance. Not only do hospital pharmacists play a central role in recognizing the need for antimicrobial stewardship at the facility level, but the pharmacy system is often the only service that can provide data on antimicrobial use before and after establishment of an ASP. In most primary care and long-term care facilities, pharmacists also play a central role in carrying out stewardship activities, with the support of physicians knowledgeable about infectious diseases. Pharmacy directors and clinical pharmacists often create the impetus to initiate and write the business cases for ASPs, because the budgets for antimicrobials and personnel to administer the programs will be affected by the content of the proposals. To this end, the Antimicrobial Stewardship and Resistance Committee of the Association of Medical Microbiology and Infectious Disease Canada (AMMI Canada) has developed a business case template for use by individual institutions that are intent on establishing an institutional ASP and acquiring funding for the required personnel.

The authors of this article, all of whom are current or former members of the AMMI Canada Antimicrobial Stewardship and Resistance Committee, represent a group of experts who have built and maintained ASPs, and the template reflects the required elements of a business case. The intent of the business case itself is to secure necessary resources from health care administrators to support ASPs in a meaningful and long-term fashion at health care institutions. The document supporting the business case template consists of an executive summary, 3 textual sections providing detailed background information to support the request, and a spreadsheet-based template for a business case analysis.

The first section of the document provides details on both the benefits of antimicrobials and the unintended consequences of these medications. For example, most executives will likely not be aware of the unique aspects of antimicrobials and their broader long-term impact on society through the spread of drug resistance.

The second section elaborates on the issues of the burden and costs of antimicrobial-resistant organisms and $C$. difficile illness. Many of the data in this section come from the 2016 report of the Public Health Agency of Canada outlining the burden of antimicrobial-resistant organisms in Canada. ${ }^{10}$ Bacteria specifically described in that report include methicillin-resistant Staphylococcus aureus, vancomycin-resistant Enterococcus, Enterobacteriaceae that are resistant to cephalosporins, and C. difficile. The language in this section is scientific and factual, but concepts such as "increased length of stay" should be readily understood by hospital administrators. Institution-specific data obtained from an institution's microbiology laboratory or from local infection prevention and control programs can be inserted within this section of the template.

The third section explores evidence linking the existence of robust ASPs with the control of antimicrobial use. ${ }^{2}$ For example, the use of antimicrobials usually drops by one-fifth following implementation of an ASP. The third section also addresses the issue of staffing, drawing from the staffing model for infection prevention and control and from provincial working groups. On the basis of these sources, the business case recommends 1.0 physician, 3.0 pharmacist, 0.5 administrative support, and 0.4 data analyst full-time equivalents per 1000 acute care beds.

A subsection also deals with the ethical responsibility of health care personnel toward their patients. ${ }^{11,12}$ More specifically, if antimicrobial use continues without restraint, many patients will experience failure of empiric therapy and perhaps suffer consequences.

The fourth (stand-alone) section is the spreadsheet-based template for a business case analysis in Microsoft Excel 2013 (Microsoft Corp, Redmond, Washington), which allows users to insert the costs and benefits of the proposed business case for their local institution. The spreadsheet file includes instructions for using the template and suggestions for determining operating budgets, estimating the costs of personnel and antimicrobials, and performing a return-on-investment analysis. Although primarily 
targeted to acute care institutions, the AMMI Canada business case for an ASP can be modified for cancer care, rehabilitation, and complex continuing care institutions.

Both the supporting Word document and the Excel business case template are available as open access resources from the AMMI Canada website (https://www.ammi.ca/?ID=126).

The need and hence the justification for antimicrobial stewardship in Canadian health care institutions is undeniable and pressing. The AMMI Canada business case for stewardship provides an easily customizable tool that individual institutions can use in seeking funding for and establishing successful ASPs. More generally, it is also intended as a call to action to promote appropriate use of antimicrobials in Canadian facilities and institutions.

\section{References}

1. Valiquette L, Cossette B, Garant MP, Diab H, Pépin J. Impact of a reduction in the use of high-risk antibiotics on the course of an epidemic of Clostridium difficile-associated disease caused by the hypervirulent NAP1/027 strain. Clin Infect Dis. 2007;45 Suppl 2:S112-21.

2. Baur D, Gladstone BP, Burkert F, Carrara E, Foschi F, Döbele S, et al. Effect of antibiotic stewardship on the incidence of infection and colonisation with antibiotic-resistant bacteria and Clostridium difficile infection: a systematic review and meta-analysis. Lancet Infect Dis. 2017;17(9):990-1001.

3. Campbell TJ, Decloe M, Gill S, Ho G, McCready J, Powis J. Every antibiotic, every day: maximizing the impact of prospective audit and feedback on total antibiotic use. PLoS One. 2017;12(5):e0178434.

4. Nault V, Pepin J, Beaudoin M, Perron J, Moutquin JM, Valiquette L. Sustained impact of a computer-assisted antimicrobial stewardship intervention on antimicrobial use and length of stay. J Antimicrob Chemother. 2017; 72(3):933-40.

5. Morris AM. Antimicrobial stewardship programs: appropriate measures and metrics to study their impact. Curr Treat Options Infect Dis. 2014;6(2):101-12.

6. Lowe CF, Payne M, Puddicombe D, Mah A, Wong D, Kirkwood A, et al. Antimicrobial stewardship for hospitalized patients with viral respiratory tract infections. Am J Infect Control. 2017;45(8):872-5.

7. Vaisman A, McCready J, Hicks S, Powis J. Optimizing preoperative prophylaxis in patients with reported ß-lactam allergy: a novel extension of antimicrobial stewardship. J Antimicrob Chemother. 2017;72(9):2657-60.

8. Antimicrobial stewardship. In: Required organizational practices handbook 2017. Ottawa (ON): Accrediation Canada; 2016. p. 40-1.

9. Countries should have AMR plans in place by May 2017. The Hague (Netherlands): International Pharmaceutical Federation; 2017 [cited 2017 May 5]. Available from: www.fip.org/www/index.php?page=news_ publications\&news=newsitem\&newsitem $=202$

10. Canadian antimicrobial resistance surveillance system. Report 2016. Ottawa (ON): Public Health Agency of Canada; 2016.

11. Rushton $\mathrm{CH}$. Creating a culture of ethical practice in health care delivery systems. Hastings Cent Rep. 2016;46 Suppl 1:S28-31.

12. Spellberg B, Srinivasan A, Chambers HF. New societal approaches to empowering antibiotic stewardship. JAMA. 2016;315(12):1229-30.

Nicole Le Saux, MD

Infectious Diseases, Children's Hospital of Eastern Ontario

Ottawa, Ontario

Bruce Dalton, BScPharm, PharmD

Pharmacy, Alberta Health Services

Calgary, Alberta

Kim Abbass, PharmD

Antimicrobial Stewardship and Infectious Diseases/Critical Care

Nova Scotia Health Authority

Sydney, Nova Scotia
John Conly, MD

Snyder Institute of Chronic Disease, Cumming School of Medicine University of Calgary

Calgary, Alberta

Nick Daneman, MD

Pharmacy, Sunnybrook Health Sciences Centre

Toronto, Ontario

Linda Dresser, PharmD

Pharmacy, University Health Network

Toronto, Ontario

Sergio Fanella, MD

College of Medicine, Faculty of Health Sciences

University of Manitoba

Winnipeg, Manitoba

Greg German, MD

Laboratory Medicine, Queen Elizabeth Hospital

Charlottetown, Prince Edward Island

Jennifer Grant, MD

Department of Pathology and Laboratory Medicine

Vancouver General Hospital

Vancouver, British Columbia

Yoav Keynan, MD

Internal Medicine, Medical Microbiology and Communicable Diseases University of Manitoba

Winnipeg, Manitoba

Tim T Y Lau, PharmD

Pharmaceutical Sciences, Vancouver General Hospital

Vancouver, British Columbia

Jamie McDonald, PharmD

Pharmacy Department, Children's Hospital of Eastern Ontario

Ottawa, Ontario

Caroline Nott, MD

Division of Infectious Diseases

The Ottawa Hospital, Civic Campus

Ottawa, Ontario

David Patrick, MD

BC Centre for Disease Control

Vancouver, British Columbia

Yvonne Shevchuk, PharmD

University of Saskatchewan

Saskatoon, Saskatchewan

Daniel Thirion, PharmD

Faculté de pharmacie, Université de Montréal

Montréal, Quebec

Andrew Morris, MD

Mount Sinai Hospital, University Health Network

Toronto, Ontario

The authors are current or former members of the Antimicrobial Stewardship and Resistance Committee of the Association of Medical Microbiology and Infectious Disease Canada who were involved in developing the business case template for antimicrobial stewardship programs described in this article.

Competing interests: John Conly has served as an unpaid Board member for the Canadian Foundation for Infectious Disease; has received personal fees from Pfizer for participation in a vaccine meeting; has received grants from the Canadian Institutes for Health Research, Alberta Innovates Health Solutions, and Pfizer; and has received support from bioMérieux to attend a hospital infections meeting and to present a lecture, all for activities outside the work of this article. Daniel Thirion has received grants from Merck Frosst Canada and Sunovion and speakers' honoraria from Merck Frosst Canada and Accelerate Diagnostics, all for activities outside the work of this article. Andrew Morris receives salary support for antimicrobial stewardship, which is the topic of this article. No other competing interests were declared. 